\title{
A Novel Technique to Instrument Proximal and Distal Adjacent Segment Pathology
}

\author{
Arvind Gopalrao Kulkarni ${ }^{1}$, Shumayou Dutta ${ }^{2}$ \\ ${ }^{1}$ Consultant Spine Surgeon, Mumbai Spine Scoliosis and Disc Replacement Centre, Mumbai, \\ ${ }^{2}$ Clinical Spine Fellow, Bombay Hospital \& Medical Research Centre, India
}

Corresponding Author: Arvind Gopalrao Kulkarni, MD, MS, FCPS, SICOT

Consultant Spine Surgeon, Mumbai Spine Scoliosis and Disc Replacement Centre, 2nd floor Room No. 206 New Wing, Bombay Hospital \& Medical Research Centre, 12, New Marine Lines, Mumbai- 400020, India

Tel: $+91-9892875490$

Fax: +91-22-22080871

E-mail: drarvindspines@gmail.com

Received: October 25, 2016

Revised: March 4, 2017

Accepted: March 9, 2017
Objective: Revision surgeries for clinical adjacent segment pathology (ASP) are fraught with challenges. While various aspects of ASP is extensively covered in literature, there is a dearth of information related to the challenges encountered during these revision surgeries and salvage options. In this technical report, the authors describe a novel surgical technique for the management of Clinical ASP. Methods: A 55 year old, obese diabetic woman, operated 5 years back for a L3-5 decompression and an instrumented postero-lateral fusion (PLF), developed symptomatic vertical instability at L5-S1 as well as lumbar canal stenosis at L2-3. Decompression and extension of the fusion both proximally and distally was planned. Several challenges were identified both technical and patient related and a novel technique developed to stabilise her. L5-S1 trans-foraminal lumbar inter-body fusion (TLIF) and L2-3 decompression and postero-lateral fusion (PLF) was fixed by bypassing the old fixation with proximal and distal screws and connecting rods. Results: Patient was subsequently mobilised out of bed the next day and continues to do well 1 year post operatively as evidenced by an improved VAS and ODI score. Conclusion: This technical report describes the current challenges encountered during revision surgery for ASD as well as the steps of a simple, easily implementable and hassle free solution to this unique problem.

Key Words: Spinal stenosis, Spinal instrumentation, Pedicle screws, Revision spine surgery, Spinal fusion

\section{INTRODUCTION}

Adjacent segment pathology has becomes a fairly common phenomenon with $5.2-18.5 \%$ of patients developing clinically significant ASP requiring surgery at 5 years ${ }^{2,8}$. Several review articles and level III and IV studies are published that discuss the etiology and risk factors for the development of ASP ${ }^{1,5.7)}$. Less often discussed are the challenges faced in performing these revision surgeries. The patient studied here is an obese, diabetic lady of African origin suffering from back and leg pain. She was operated 5 years back for a L3-5 decompression and an instrumented postero-lateral fusion (PLF). Her examination and imaging revealed clinical ASD both superiorly and inferiorly to the fusion mass. While she had developed a lumbar canal stenosis at L2-3, she also developed a vertical instability at L5-S1 (Fig. 1). Her VAS back/leg was $8 / 10$ and $9 / 10$ respectively and her ODI was 67.2. No prior records of her implant system was available. In this paper the authors describe a novel technique developed to instrument the proximal and distal extension of fusion as well as discuss the challenges commonly encountered during revision ASP.

\section{TECHNIQUE}

\section{Positioning and Anaesthesia}

General anaesthesia was administered. Patient was placed prone on parallel bolsters with the abdomen hanging free.

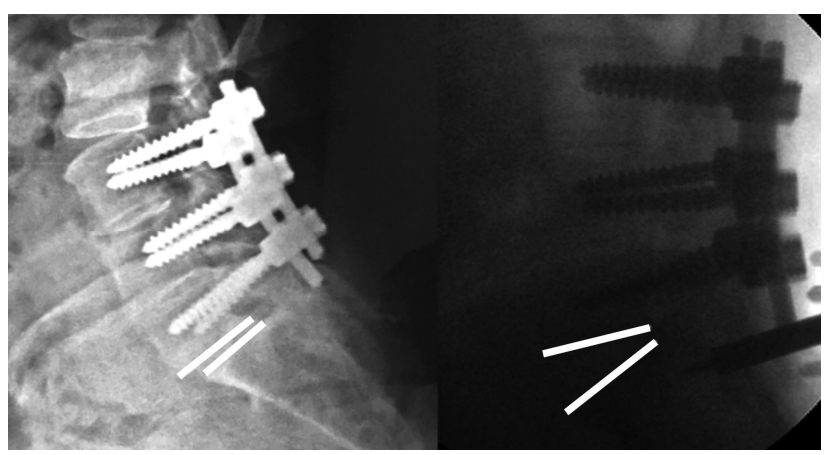

Fig. 1. Clinical ASP (vertical instability) developed at L5-S1, 5 years after index surgery. 


\section{Surgery}

A midline incision taken from L2 to S2 and subcutaneous tissue dissected till the muscle facia was exposed through the entire length of the incision. Sub-periosteal exposure of the L2-L3 and L5-S1 level achieved in the conventional manner. The L2-L3 exposure was continued para-spinally using manual palpation of the implant as a guide. Implant was exposed adequately. Inferiorly this exposure was continued with the L5-S1 sub-periosteal dissection. Care was taken to not violate the superior (L1-2) Supra/Inter-spinous ligaments and also the superior (L2-3) facet joint capsule.

Several attempts were made to remove the implants using instruments of at least 5 implant companies. Failure to remove the rods, forced an alternative strategy.

Extra-capsular entry for both L2 pedicle screws marked, probed and cannulated. Similarly bilateral S1 screws entry prepared, probed and cannulated. Depth measured and a screw length of $10 \mathrm{~mm}$ in excess of that measured was chosen both for L2 and S1. The tulips were kept $10 \mathrm{~mm}$ above the facet so as to

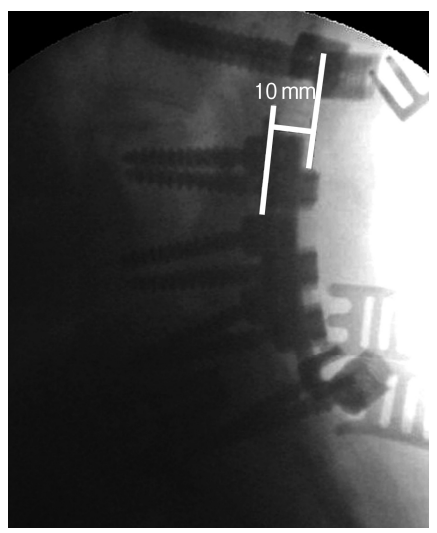

Fig. 2. Tulips are kept $10 \mathrm{~mm}$ superficial than the prior fusion tulips depth.

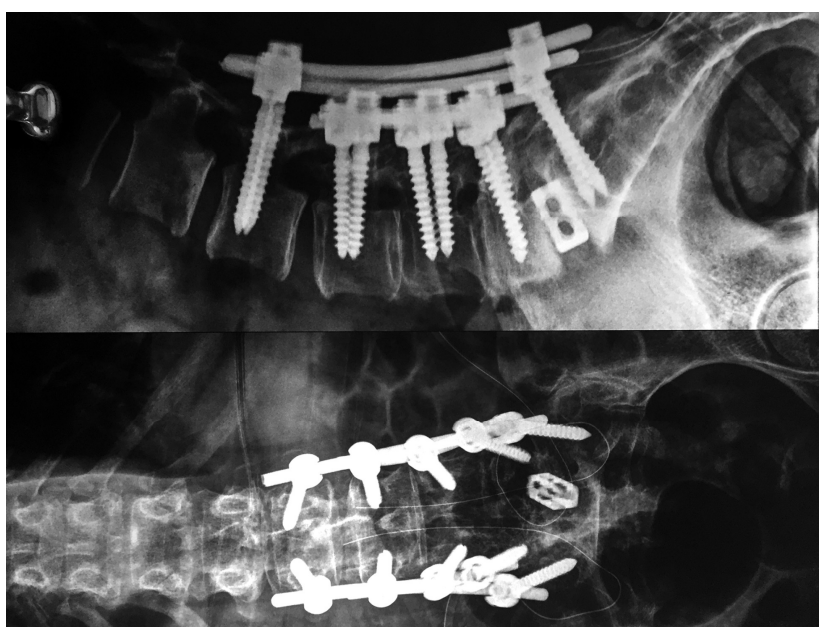

Fig. 3. Immediate post-operative image showing AP and Lateral of LS Spine showing the bridging fixation. be at a depth more superficial than the previous implant construct (Fig. 2). L5-S1 right facetectomy and disc space prepared for the interbody cage placement and fusion. L2-3 decompressed and graft placed postero-laterally. L2 to S1 distance measured and a connecting rod with the same lordosis as the previous construct placed, by-passing the entire previous fusion mass. Inner nuts were fixed onto the $\mathbf{L}$ screw. For compression between the two screws a vice-grip was fixed onto the rod close to the S1 screw. A compressor was then used to compress between the vice-grip and the S1 screws. After adequate compression, the inner nuts were tightened for the final time. Final imaging confirmed satisfactory implant placement (Fig. 3).

Sutures were taken over a drain.

\section{RESULTS}

Operative time was 174 mins and there was a blood loss of $230 \mathrm{~mL}$. The patient was mobilised out of bed the next day and discharged on the post-operative day 4. There was no implant prominence and wound healing was un-eventful. Her VAS back/leg improved to $3 / 10$ and $2 / 10$ respectively with an ODI of 24.2 at 1 year follow up (Fig. 4).

\section{DISCUSSION}

Various treatment options for clinical ASP are described in literature. These range from decompression, extension of fusion and even total disc arthroplasty ${ }^{3}$. Less often described are the unique set of challenges these surgeries are fraught with. Traditionally, extension of fusion involves removing the inner nut and the connecting rod, instrumenting the involved adjacent vertebra with a pedicle screw and placing a new connecting rod and inner nut or if this fails then using a rod to rod (domino) connector to clasp a new short rod onto the old construct. All this is done after completing the decompression and fusion

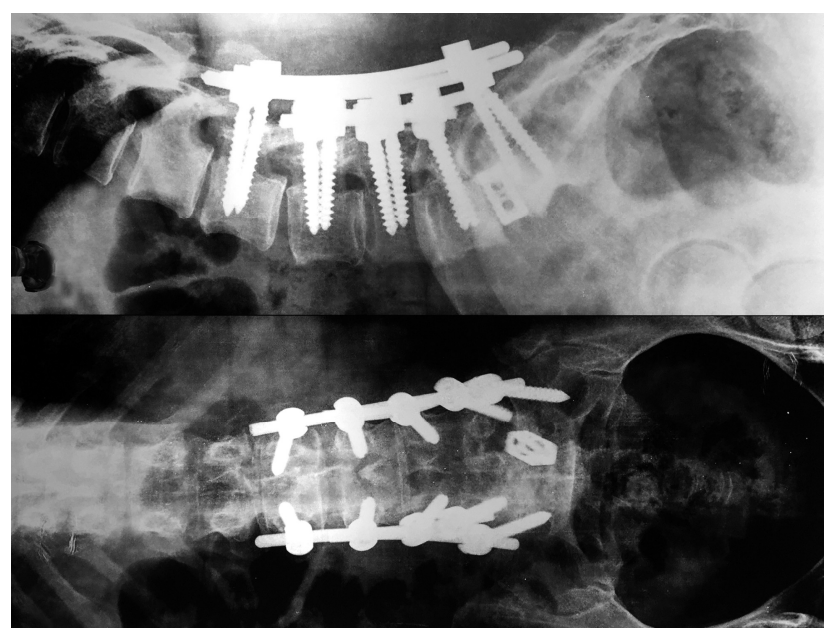

Fig. 4. One year post-operative X-Ray image of the lumbar spine. 
specific work (interbody preparation for PLIF/TLIF or postero-lateral grafting for a PLF). With the rise in the rates of spinal arthrodesis offered, there has been a simultaneous growth in the number of implant companies producing their variation of the pedicle screw rod system in the market ${ }^{10)}$. In addition to this, the design evolution of these implants have resulted in many companies discarding the previously used instrument set for a newer system. Poor medical record keeping may also result in the inability to identify the implant system correctly leaving a lot to guess work based on the X-ray images. With over 25 indigenous companies manufacturing implants in India, trial and error intraoperatively to remove the inner nut with different drivers is not a feasible option. Such situations are often encountered and result in the surgeon resorting to more radical attempts to remove the rods and screws. It is a common practise to break the inner nut and remove the connecting rod to replace it with a longer one that spans the new screw. However, even the connecting rods of each implant systems differ subtly in the diameter. While some companies make connecting rods of the lumbar spine with a diameter of $5.5 \mathrm{~mm}$, others use 6 $\mathrm{mm}$. This subtle difference is enough to prevent the new rod from sitting snugly in the tulip. Also, this variation in the implant design also makes rod to rod (domino) connectors from other companies invalid. Newer designs of lateral connectors are also available which clasp onto the old construct and have a slot for a new rod to be added for only the proximal/distal screws. These however are difficult to apply if there is crowding of the tulips of the old construct leaving not enough space for the connectors. These are also not widely available in many regions of the country. If all the above fail, surgeons resort to replacing the entire implant system with a new one. Screw-drivers also lack uniformity making screw removal difficult. Applying excess axial stress while removing a screw with an ill-fitting screw driver/plier can lead to breakage of the bony ridges between the screw threads. As the last resort, the screw can be removed by breaking the rod close to the tulip using a jumbo cutter and de-rotating the screw rod system to remove the screw ${ }^{4)}$. Even though this is feasible for a single level fusion, executing it in a multilevel fusion is extremely challenging and time consuming. As can be imagined, these are very stressful, frustrating and un-predictable. In addition to this, long surgeries in patients with co-morbidities increases the chances of adverse outcomes such as infection, ${ }^{6,9}$. The technique mentioned above is unique as all these implant related issues can be avoided, decreasing the probability of a negative outcome. There was no implant prominence and in this situation the patients' obesity worked to an advantage. It is probably advisable to avoid this procedure in a thin patient as wound closure and implant prominence may be an issue. As an extension, this surgery can also be performed minimally invasively with newer versions of the multi-level sextant system for guiding the new rod into the tulips, with due caution.

\section{CONCLUSION}

In this technical report, we discuss the challenges faced in extending fusion for a clinical ASD and provide a simple, easily implementable and hassle free solution. This fusion bridging technique also reduces operative time and the concurrent risks of prolonged surgery. Knowledge of this 'bail-out' technique will provide confidence to surgeons attempting revision surgeries.

\section{REFERENCES}

1. Cho KJ, Suk SI, Park SR, Kim JH, Kim SS, Choi WK, et al: Complications in posterior fusion and instrumentation for degenerative lumbar scoliosis. Spine 32:2232-2237, 2007

2. Daniel C.N, Joseph R.D, Andrea C.S, Daniel R, Jens R C and Paul A. A: Methodology for the Systematic Reviews on an Adjacent Segment Pathology. Spine 37(22S):S10-S17, 2012

3. Dean C, Mark D, Jeff $H$ and Del CN: The Treatment of Lumbar Adjacent Segment Pathology After a Previous Lumbar Surgery, A Systematic Review. SPINE 37(22S):S180-S188, 2012

4. Kamil C, Cengiz I, Levent A, Ali A, Mustafa O: Easy retrieval of polyaxial tulip-head pedicle screws by " $U$ " rod technique. European Spine Journal 19(1):144-146, 2010

5. Kumar MN, Baklanov A, Chopin D: Correlation between sagittal plane changes and adjacent segment degeneration following lumbar spine fusion. Eur Spine J 10:314-319, 2001

6. Margaret A, Jennie M, Carl L, Louis B, Marilyn J, Joshua V, et al: Risk factors for surgical site infection in spinal surgery, Journal of Neurosurgery. Spine 98(2):149-155, 2003

7. Min JH, Jang JS, Jung B, Lee H, Choi W, Shim C, et al: The clinical characteristics and risk factors for the adjacent segment degeneration in instrumented lumbar fusion. J Spinal Disord Tech 21:305-309, 2008

8. Park P, Garton HJ, Gala VC, Hoff, Julian T, McGillicuddy, et al: Adjacent segment disease after lumbar or lumbosacral fusion: review of the literature. Spine 29:1938-1944, 2004

9. Pull T, Albert F, Cohen, David B:. Incidence, Prevalence, and Analysis of Risk Factors for Surgical Site Infection Following Adult Spinal Surgery: Spine 34(13):1422-1428, 2009

10. Weinstein JN, Lurie JD, Olson PR, Bronner KK, Fisher ES: United States' trends and regional variations in lumbar spine surgery: 1992-2003. Spine 31:2707-2714, 2006 